\title{
Superconducting super motor and generator
}

\author{
Minaru Kawamura (河村実生), and Jonathan A. Jones
}

\begin{abstract}
We have developed a new type of superconducting synchronous rotating machine whose self-induction is cancelled out, with the intention of achieving a very high power-to-weight ratio including the weight of the cooling system. Magnetic cores are used to direct the magnetic field from permanent magnets on the rotors onto superconducting wires on the stator, and the reaction of the Lorenz force is used to drive the rotors. Cancellation of self-induction in the cores enables the elimination of core-losses and magnetic saturation, permitting the core mass to be reduced significantly, and also reducing ac losses in the superconducting wires. In this work a prototype prepared using $100 \mathrm{~m}$ of second generation high temperature superconductor (2G-HTS) wire is described, and its characteristics are measured and compared with a numerical simulation. We conclude that electrical rotating machines with power-to-weight ratios comparable to jet engines could be developed with 2G-HTS wire.
\end{abstract}

Index Terms-High-temperature superconductor, motors, generators, rotating machines

\section{INTRODUCTION}

$\mathrm{S}$ INCE the discovery of Type II superconductors, especially niobium-tin, much effort has been devoted to large scale applications. Useful superconducting power devices have been developed [1], in particular for producing strong dc magnetic fields. As yet, however, ac losses in superconductors have made it difficult to create practical and useful fully superconducting rotating machines, and these losses are considered to limit their effectiveness. Perhaps the greatest advancements to date have been in the area of low rotation speed ship propulsion motors, where a power-to-weight ratio of about $0.5 \mathrm{~kW} / \mathrm{kg}$ has been reported with a rotor wound from high temperature superconductor. This is an improvement by a factor of about five compared to a conventional motor, even though conventional copper air-core windings were employed on the stator, and a cryogenic cooling system, based on gaseous helium, is required [2], while several new designs and approaches are currently in progress $[3,4,5]$.

It appears, however, that 2G-HTS wire [6,7], which permits a high current density with strong magnetic fields, enables the development of rotating machines with very larger power-toweight ratios. Consider, for example, a current of $100 \mathrm{~A}$ in $1,000 \mathrm{~m}$ of $2 \mathrm{G}-\mathrm{HTS}$ wire under a magnetic field of $1 \mathrm{~T}$ : this will induce a Lorentz force of 100,000 N, and if this force were used in a rotating machine with a rotor diameter of $1 \mathrm{~m}$

This work was supported in part by the Japan MEXT-Supported Program for the Strategic Research Foundation at Private Universities, 2012-2016.

Minaru Kawamura is with the Department of Electrical and Electronic Engineering, Okayama University of Science, Okayama, 700-0005, Japan (email:minaru@ee.ous.ac.jp)

Jonathan A. Jones is with the Department of Physics, University of Oxford, Oxford OX1 3PU, U.K. (e-mail: Jonathan.Jones@ qubit.org) rotating at a speed of 3,600 rpm, it would generate a power of about $19 \mathrm{MW}$. Assuming a copper/Hastelloy tape width of 4 $\mathrm{mm}$ and a thickness of $0.1 \mathrm{~mm}$, the volume of the wire is only about $400 \mathrm{~cm}^{3}$ (for the superconductor itself, only $4 \mathrm{~cm}^{3}$ ), and the weight is less than $3.6 \mathrm{~kg}$. This indicates a limiting powerto-weight ratio of $5,000 \mathrm{~kW} / \mathrm{kg}$, much higher than the 160 $\mathrm{kW} / \mathrm{kg}$ achieved by the HPFTP turbopump in the Space Shuttle main engine [8].

Many practical problems remain of course: how to apply the magnetic field; how to convert the Lorentz force to torque; how to efficiently refrigerate the superconductor; the effects of self-inductance and ac losses in the superconducting wire, and so on. Here a novel design of superconducting rotating machine in which self-induction is cancelled out is proposed to address all these problems: in effect this design combines the advantages of iron and air cores while avoiding their disadvantages. Iron cores enable enhanced control of magnetic flux, allowing increased efficiency and reduced ac-losses, but they are heavy, have core losses and magnetic saturation problems. Air cores are light and do not suffer from losses or saturation, but lack the enhanced flux control. We call this machine a super motor/generator to distinguish it from conventional superconducting rotating machines. A first prototype has been constructed to demonstrate the operating principles, and magnetic field simulations have been made and compared with experimental data. These initial results suggest that super motors could be constructed with high efficiency and power-to-weight ratios comparable to jet engines used in aircraft.

\section{STRUCTURE AND FEATURES OF THE SUPER MOTOR/GENERATOR}

The structure of the prototype super motor/generator is schematically illustrated in Fig. 1. The two rotors and the

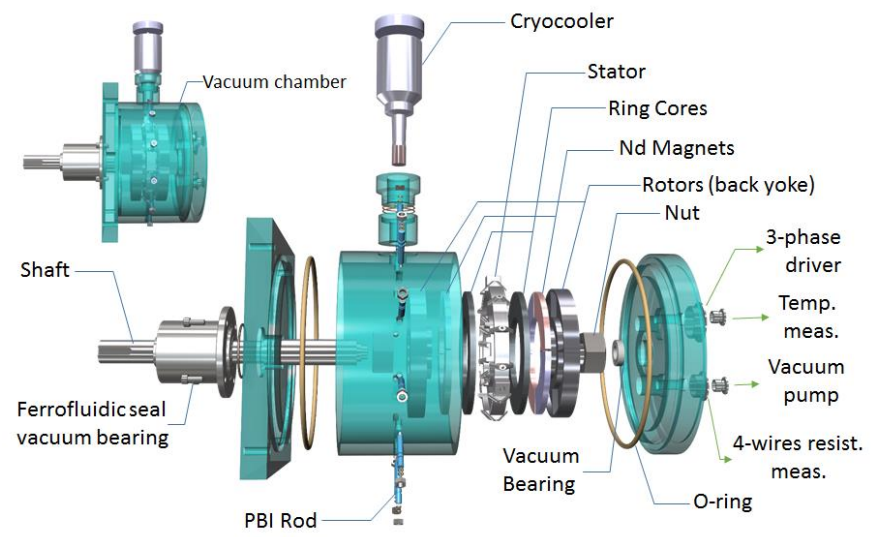

Fig. 1. Schematic diagram of the prototype super motor/generator which is a synchronous four-pole machine. The left hand rotor is the mirror image of the right hand rotor, but is partly hidden by the vacuum chamber. 

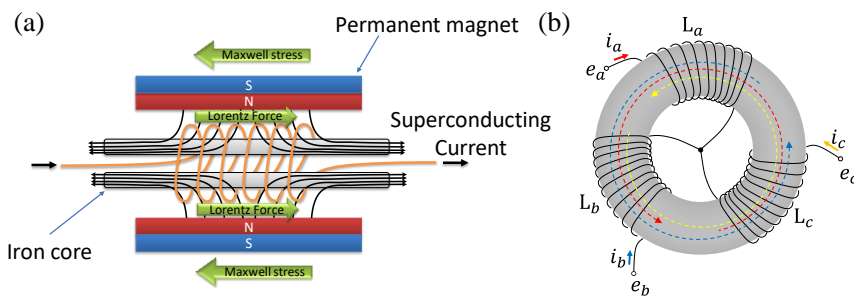

Fig. 2. (a) Principle of torque generation in the super motor. (b) Schematic diagram of the armature coils. In the prototype each branch is wound as two separate coils of 90 turns each, on opposite sides of the stator and connected in series to give a six-pseudopole stator.

stator are placed in a single vacuum chamber, and the shaft is supported with ball bearings shielded with ferrofluid to maintain a high vacuum. Each of the rotors is composed of four Neodymium permanent magnets with a back yoke, that is, it has four poles, with a diameter of about $25 \mathrm{~cm}$. The stator between the rotors is supported by 12 rods of polybenzimidazole (PBI) for thermal insulation. The stator itself is made of metal for good thermal conductivity, and contains two $10 \mathrm{~mm}$ thick toroidal cores wound from silicon iron tape, one on each side. The superconducting 2G-HTS wires are wound on the stator, and then impregnated with cryogenic epoxy resin. This stator is directly refrigerated by a compact cryocooler, and wrapped with superinsulation composed of 20 layers of polyester foil, aluminized on one side in $1 \mathrm{~cm}$ squares, interleaved with polyester spacer material. The total length of superconducting wire used is $100 \mathrm{~m}$, with a tape width of $2 \mathrm{~mm}$ and a thickness of $0.1 \mathrm{~mm}$. The thickness of the yttrium based superconductor itself is only $1 \mu \mathrm{m}$, while the tape base is made principally of copper and a nickel-tungsten nonmagnetic super alloy. The wide gap between the stator and rotors (for this prototype, $20 \mathrm{~mm}$ ) easily contains the wire and insulation, permitting the wire length to be extended up to $1 \mathrm{~km}$.

In this super motor/generator, the cores play two crucial roles. Firstly they efficiently apply the magnetic field to the superconducting wires to generate a Lorentz force, whose reaction causes torque, as depicted in Fig. 2 (a), while avoiding applying magnetic fields to the metallic stator body, which would induce eddy currents. The flux from the north pole of the permanent magnets penetrates perpendicularly to the core, eventually leaving perpendicular to the edges of the core to reach the neighbouring south poles. Secondly by making the mutual inductance almost equal to the self inductance they enable the cancellation of the self-induction produced in the cores by the superconducting currents. This

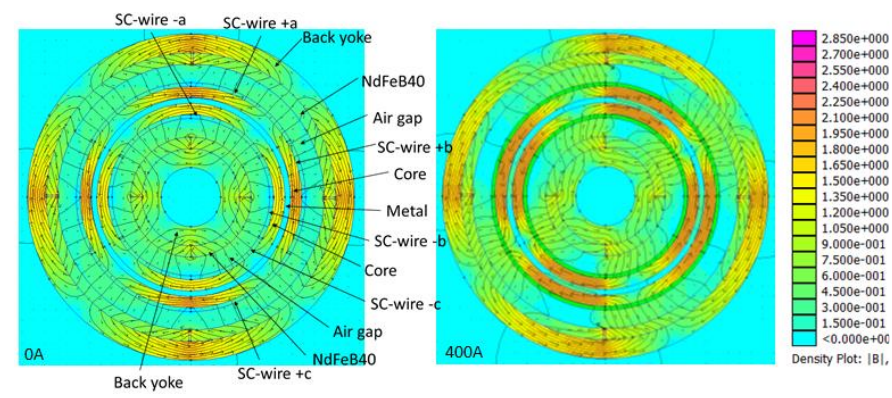

Fig. 3. Magnetic flux distribution in the axial type super motor for currents of 0 and $400 \mathrm{~A}$, where 90 turns/section. The colour scale runs from 0 to $3 \mathrm{~T}$. cancellation gives several remarkable features: the elimination of inductance enables a perfect power factor and high speed operation; prevention of magnetic saturation in the cores allows reduction of their weight; and ac losses in the superconductor due to the self-field are significantly reduced.

This cancellation is achieved by connecting the superconducting coils on the armature to each other as shown in Fig. 2 (b), so that the total induction generated in the cores by the currents in any coils is almost cancelled out through mutual-inductances. Assuming that the three inductors are equivalent, that is they have same self-inductance, $L_{0}$, the mutual-inductance will be $L_{0}-\Delta L$, differing from the selfinductance only due to flux leakage, which is greatly reduced by the cores. The circuit equations can be written in matrix form as,

$$
\left[\begin{array}{l}
e_{a} \\
e_{b} \\
e_{c}
\end{array}\right]=\frac{d}{d t}\left[\begin{array}{ccc}
L_{0} & L_{0}-\Delta L & L_{0}-\Delta L \\
L_{0}-\Delta L & L_{0} & L_{0}-\Delta L \\
L_{0}-\Delta L & L_{0}-\Delta L & L_{0}
\end{array}\right]\left[\begin{array}{c}
i_{a} \\
i_{b} \\
i_{c}
\end{array}\right]=\frac{d}{d t}\left[\begin{array}{ccc}
\Delta L & 0 & 0 \\
0 & \Delta L & 0 \\
0 & 0 & \Delta L
\end{array}\right]\left[\begin{array}{c}
i_{a} \\
i_{b} \\
i_{c}
\end{array}\right],
$$

where the second form can be obtained from the first as the currents sum to zero and so any constant can be added to the matrix. The effective inductance is then given by the leakage inductance, $\Delta \mathrm{L}$, which is expected to be very small relative to the self-inductance for toroidal cores.

\section{THE SIMULATION}

The magnetic field in the super motor has been calculated using finite element methods to estimate the torque and the power output which can be expected. In this simulation an equivalent cylindrical 2D model is used for convenience, instead of the actual 3D disk structure. Fig. 3 shows the magnetic fields for three-phase currents of amplitude 0 , and $400 \mathrm{~A}$, at zero degrees phase. It can be seen that the symmetry of magnetic field is broken as the current flow is increased. A torque acts on the permanent magnets as a reaction to the Lorentz force caused by the currents and the magnetic field, which can be represented as a Maxwell stress force.

Fig. 4 shows calculated torques and torque distortions as a function of current for three different core materials: silicon iron steel, ferrite, and air. Calculations for an eight-pole machine with $1 \mathrm{~km}$ of superconducting wire are also shown in the Fig.. For air cores the torque is directly proportional to the current, but the clear curvature for iron and ferrite cores arises from magnetic saturation effects. The calculated torque distortions are also primarily caused by partial magnetic saturation. Note that the distortions for eight-pole machines are much less than those for four-pole as a result of the reduced distance between poles.
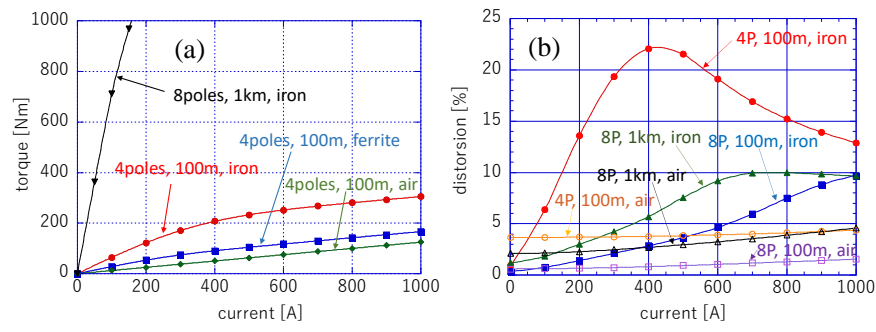

Fig. 4. (a) Calculated torque and (b) torque distortion as a function of current for various machine models. The red curve ( 4 poles, $100 \mathrm{~m}$, iron core) corresponds to the prototype described here. 


\section{EXPERIMENTAL RESULTS}

A prototype was constructed and tested in our laboratory as shown in Fig. 5. The super motor/generator was connected to a conventional induction motor through a torque meter.

Power generation characteristics were measured with the super motor driven for a short time by a normal motor to eliminate temperature dependences (for example, the losses in the vacuum bearing depend strongly on the temperature of the ferrofluid and the superconducting armature). The open-circuit electromotive force and the voltage drop across an external 1 $\Omega$ load are shown in Fig. 6 (a) as a function of frequency. By fitting these results to the function $\alpha \omega R / \sqrt{R^{2}+\omega^{2} L^{2}}$, where $\alpha$ is an amplitude, $R=1 \Omega$ is the external load, $\omega$ is angular frequency, and $L$ is the residual inductance, the leakage inductance can be estimated at $2.2 \mathrm{mH}$. The measured result is four times larger than expected from simulations, perhaps because the superconducting wire is not wound neatly enough.

Fig. 6 (b) shows the generated output power (calculated from the voltage across the $1 \Omega$ external load) and the consumed input power (determined using the torque meter) as a function of driving frequency. The sum of the open-circuit input power and the power output is consistent with the loaded input power, indicating that self-field ac losses in the superconducting wires are almost negligible, as expected due to the cancelation of self-induction. The good fits indicate that experimental torques, which can be calculated from the generated output power and the driving frequency, are almost proportional to current, as expected from the red curve in Fig. 4; we were unable to explore larger currents where significant curvature is expected to appear.

On open circuit most of the input power is consumed by friction in the ferrofluid vacuum bearing but ac-losses due to the rotating magnetic field are also included. These two components can be distinguished using a thermal method, as the ac-losses will be converted to heat in the stator, which is thermally isolated from the rest of the motor/generator assembly. Fig. 7 (a) shows how the temperature of the cold head changes as the driving speed was slowly changed between $300 \mathrm{rpm}$ and zero, with the super generator on open circuit.

Thermal equilibrium cold head temperatures, $\theta$, were estimated as averages of the final temperatures from the

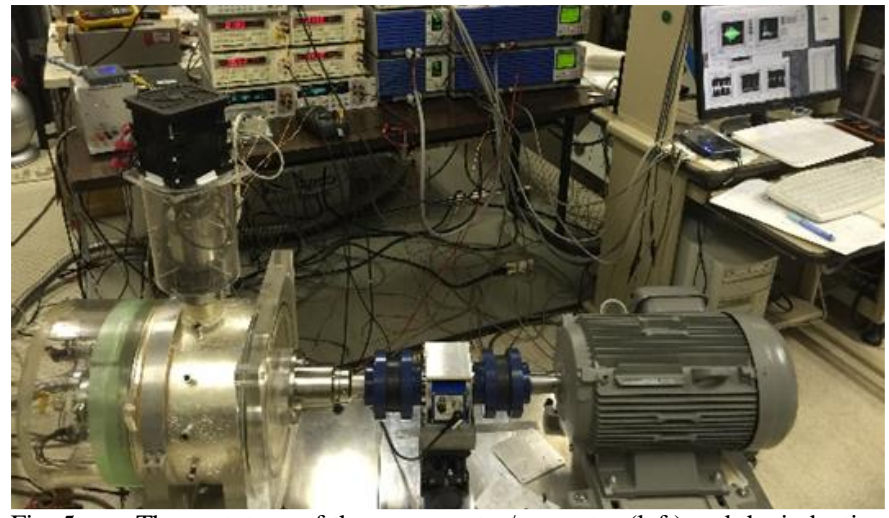

Fig. 5. The prototype of the super motor/generator (left) and the induction motor (right) used for test driving.
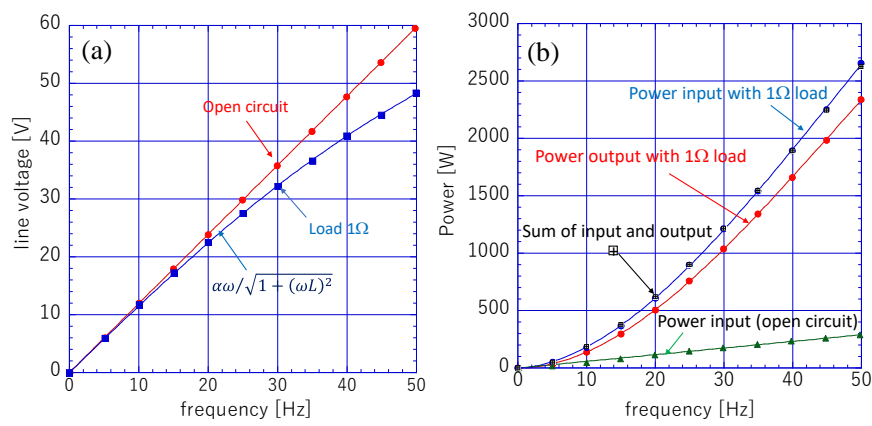

Fig. 6. (a) Open circuit emf and voltage drop across a $1 \Omega$ load (Y-connection) as a function of frequency, which is twice the frequency of the driving motor as the generator has four rotor-poles. (b) Comparison of power output and input for open and $1 \Omega$ loaded circuits. The sum (black crosses) of the opencircuit power input (green triangles) and the output on the $1 \Omega$ loaded circuit (red circles) is almost equal to the power input on the $1 \Omega$ loaded circuit (blue circles), indicating that ac losses from self-induction are negligible.

falling and rising curves, and are plotted in Fig. 7 (b). Since the whole coil, which has a transition temperature of $92 \mathrm{~K}$, transits sharply to the superconducting state at cold head temperatures between $83 \mathrm{~K}$ and $85 \mathrm{~K}$, we estimate that the temperature in the coil exceeds the cold head temperature by about 7 to $9 \mathrm{~K}$. Assuming the Bean model, with the externally applied field much larger than the full penetration field so that the critical current is proportional to $\left(T_{c}-\theta\right)$, the temperature below the critical temperature $T_{\mathrm{c}}$, the ac losses are given by $Q(\theta)=a f\left(T_{c}-\theta\right)[9,10]$, where $f$ is the ac frequency in

Hz. The cooling capacity of the cryocooler at constant input power is approximately linear near its limiting temperature $\theta_{0}$, and can be described by $C(q)=b\left(q-q_{0}\right)$. Combining these two equations leads to a thermal equilibrium temperature, $\theta_{e q}(f)=\left(f T_{c}+r \theta_{0}\right) /(f+r)$, where $r=b / a$. This provides a good fit to the experimental data, as shown in Fig. 7 (b).

From this fit and the known value of $b=0.31 \mathrm{~W} / \mathrm{K}$ we obtain $a=0.0713 \mathrm{~W} / \mathrm{Hz} / \mathrm{K}$, and so $Q(77 \mathrm{~K})=1.07 \mathrm{~W} / \mathrm{Hz}$. This value for the ac-losses is similar to a value of $1.24 \mathrm{~W} / \mathrm{Hz}$ estimated from the experimental results of Amemiya et al. [11] assuming that the amplitude of the ac magnetic field is $0.276 \mathrm{~T}$, estimated from the experimental torque.

Rotation experiments as a motor under light load were also performed with a driving circuit containing two current sources and one voltage source as shown in Fig. 8 (a), where
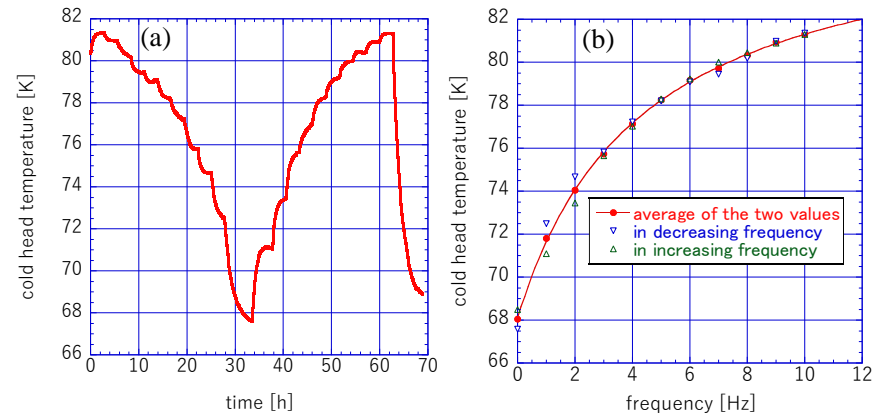

Fig. 7. (a) Changes to the cold head temperature when the rotor is driven; the rotation speed is first decreased from $300 \mathrm{rpm}$ to zero and then increased back to $300 \mathrm{rpm}$ again. (b) The cold head temperature at thermal equilibrium as a function of driving frequency. The data is well described by $\theta_{\text {eq }}(f)=\left(f T_{\mathrm{c}}+r \theta_{0}\right) /(f+r)$, with $r=4.35 \mathrm{~s}^{-1}, T_{\mathrm{c}}=87 \mathrm{~K}$, and $\theta_{0}=68 \mathrm{~K}$. 
(a)

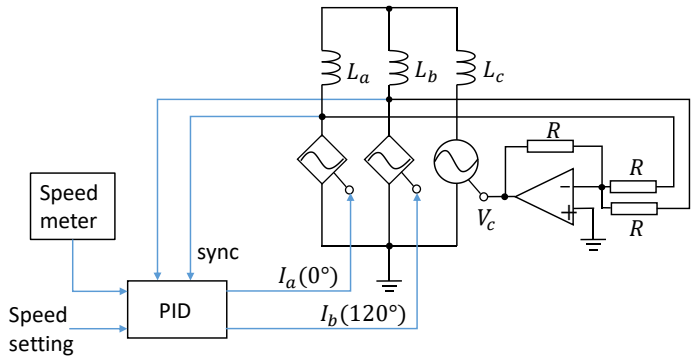

(b)
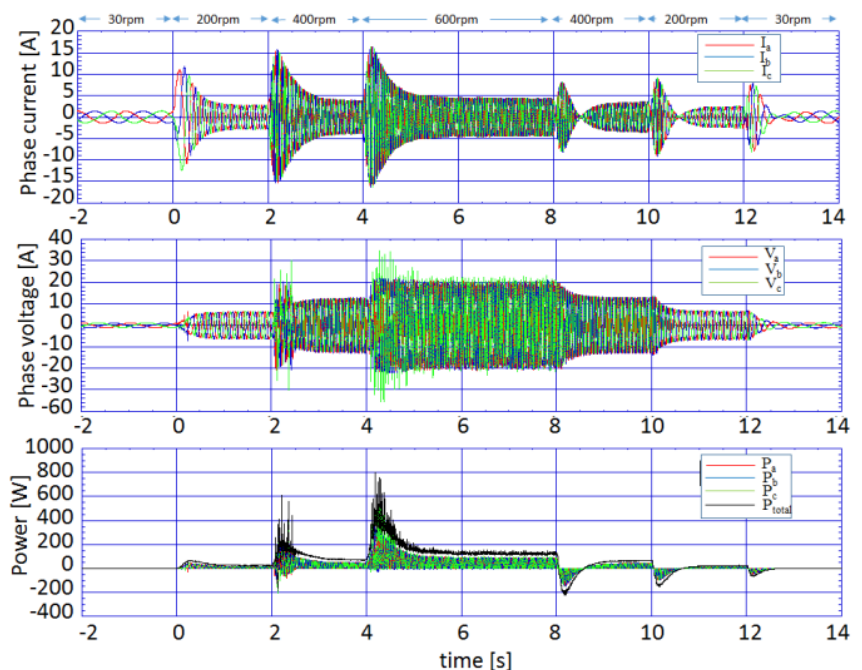

Fig. 8. (a) Driving circuit for investigating motor operation. (b) The instantaneous currents, electromotive force, and power when driving with the circuit in the figure (a). The speed is first increased in steps of approximately

the electromotive force generated on the coil from the inductance $L_{\mathrm{c}}$ is cancelled by the voltage source. The instantaneous currents, voltages and powers for the three phases are shown in Fig. 8 (b) when the rotation speed is increased to $600 \mathrm{rpm}$ in steps of about $200 \mathrm{rpm}$. From this it can be seen that the power is always positive during periods of steady driving, indicating that the currents and voltages are inphase and so the power factor is almost $100 \%$ in this current and speed range. When the rotation speed is decreased the consumed power is negative, indicating that the motor is acting as a generator, but during these short periods the power is always negative, so that the power factor is once again $100 \%$. Thus we conclude that the self-induced EMF is negligibly small relatively to the generated EMF in this experimental region. The speed range was limited by the available peak voltage $( \pm 20 \mathrm{~V})$ of the current source, but the very high observed power factor assures effective operation over a much wider frequency range.

\section{CONCLUSIONS AND DISCUSSION}

The simulations and experiments described above confirm that the prototype super motor/generator achieves the key features expected for this design. In particular the effective cancellation of inductance ensures that the ac losses are dominated by effects arising from the rotating magnetic field. The prototype achieves a power output of $2,400 \mathrm{~W}$ with ac losses of only $54 \mathrm{~W}$ at $1,500 \mathrm{rpm}$, for a nominal efficiency of $98 \%$. This figure, however, excludes the energy consumption of the cryocooler required to remove waste heat from the superconducting stator. We assume that the cryocooler requires a power equal to 15 times its cooling capacity, leading to a comprehensive efficiency of $69 \%$.

This calculation shows the importance of minimising the remaining ac losses, which arise from the rotating magnetic field, to achieve the highest comprehensive efficiency. These are proportional to the width of superconductor perpendicular to the field. As the superconducting tape is very anisotropic, with a superconducting layer thickness of only $1 \mu \mathrm{m}$, these losses could in principle be reduced by a factor of thousands by winding the coil with the tape perpendicular to the stator rather than flat against it. Perfectly perpendicular winding is not practical, but it is possible to reduce the ac losses by a factor of $\sin (\alpha)$, where $\alpha$ is the average angle between the tape and the perpendicular to the stator.

In table I, the parameters for the prototype (\#1) and design models (\#2 to \#4') of the super motor are summarized, where the wire lengths and widths, number of poles and operating temperatures are varied, and the ac-losses are estimated assuming that they are proportional to the critical current, wire length, frequency and the perpendicular component of the magnetic field. It can be seen that model \#4 in Table I achieves a power-to-weight ratio comparable to jet engines. Reducing the operation temperature to $60 \mathrm{~K}$ would double the critical current, and thus the power-to-weight ratio, but the aclosses also increase with critical current, and there is no efficiency advantage: indeed the comprehensive efficiency including power consumption in the cryocooler will get worse. A comprehensive power-to-weight ratio above $10 \mathrm{~kW} / \mathrm{kg}$, including the weight of the cryocoolers, would be possible if the transverse component of the magnetic field could be suppressed down to one-hundredth by optimizing both coil winding and magnetic structure.

\section{ACKNOWLEDGMENT}

The authors would like to thank undergraduate and graduate students of our laboratory for their contributions to the program, and Mr. Yukio Toda and Mr. Hajime Ikeda of the Toda Racing co., Ltd for supporting 3D modeling.

TABLE I

Parameters for the prototype and design models of the super motor

\begin{tabular}{lcrrrrr} 
model & Units & $\# 1$ & \multicolumn{1}{c}{$\# 2$} & $\# 3$ & $\# 4$ & $\# 4^{\prime}$ \\
\hline operation temp. & $\mathrm{K}$ & 77 & 77 & 77 & 77 & 60 \\
number of poles & - & 4 & 4 & 8 & 8 & 8 \\
wire length & $\mathrm{m}$ & 100 & 400 & 1000 & 1000 & 1000 \\
wire width & $\mathrm{mm}$ & 2 & 4 & 4 & 8 & 8 \\
rated speed & $\mathrm{rpm}$ & 3600 & 3600 & 3600 & 3600 & 3600 \\
\hline frequency & $\mathrm{Hz}$ & 120 & 120 & 240 & 240 & 240 \\
rated phase current & $\mathrm{A}(\mathrm{rms})$ & 35 & 71 & 71 & 141 & 302 \\
rated phase voltage & $\mathrm{V}(\mathrm{rms})$ & 82 & 260 & 894 & 740 & 551 \\
rated torque** & $\mathrm{Nm}$ & 23 & 146 & 503 & 833 & 1322 \\
rated power output & $\mathrm{W}$ & 8,744 & 55,181 & 189,560 & 314,023 & 498,382 \\
Power/weight $(30 \mathrm{~kg})$ & $\mathrm{kW} / \mathrm{kg}$ & 0.29 & 1.84 & 6.32 & 10.47 & 16.61 \\
\hline ac-losses $\left(90^{\circ}\right)$ & $\mathrm{W}$ & 149 & 1,190 & 5,952 & 11,904 & 25,395 \\
efficiency $\left(90^{\circ}\right)$ & $\%$ & 98.3 & 97.8 & 96.9 & 96.2 & 94.9 \\
power cons. $\left(90^{\circ}\right)$ & $\mathrm{W}$ & 2,232 & 17,856 & 89,280 & 178,560 & 639,959 \\
compreh. eff. $\left(90^{\circ}\right) *$ & $\%$ & 79.7 & 75.6 & 68.0 & 63.8 & 43.8 \\
\hline ac-losses $\left(5^{\circ}\right)$ & $\mathrm{W}$ & 13 & 104 & 519 & 1,038 & 2,213 \\
efficiency $\left(5^{\circ}\right)$ & $\%$ & 99.9 & 99.8 & 99.7 & 99.7 & 99.6 \\
power cons. $\left(5^{\circ}\right)$ & $\mathrm{W}$ & 195 & 1,556 & 7,781 & 15,563 & 33,200 \\
compreh. eff. $\left(5^{\circ}\right)^{*}$ & $\%$ & 97.8 & 97.3 & 96.1 & 95.3 & 93.8 \\
$*$ *The comprehsive efficiencies include the power consumption of the cryocooler. & \\
**The rated torque is estimated from simulation values divided by $\sqrt{2}$. & &
\end{tabular}




\section{REFERENCES}

[1] ed. H. Rogalla, and P. H. Kes, 100 years of superconductivity. New Yoke: CRC Press, 2011, Ch. 12, ISBN 9781439849460

[2] B. Gamble, G. Snitchler, and T. MacDonald, Full power test of a 36.5 MW HTS propulsion motor, IEEE Trans. Appl. Supercond., 21 (2011) 1083

[3] Moon H, Kim Y-C, Park H-J, Yu I-K and Park M, An introduction to the design and fabrication progress of a megawatt class $2 \mathrm{G}$ HTS motor for the ship propulsion application, Supercond. Sci. Technol. 29 (2016), 034009

[4] Fuger R., Matsekh A., Kells J., Sercombe D. B. T. and Guina A., A superconducting homopolar motor and generator-new approaches, Supercond. Sci. Technol. 29 (2016), 034001

[5] Marino I. et al., Lightweight MgB2 superconducting 10MW wind generator, Supercond. Sci. Technol. 29 (2016), 024005

[6] Y. Y. Xie et al., Second generation high-temperature superconducting wires for fault current limiter applications. IEEE Trans. Appl. Supercond., 17 (2007), 1981

[7] V. Selvamanickam, Y. Chen, T. Shi, Y. Liu, N. D. Khatri, J. Liu, Y. Yao, X Xiong, C. Lei, S. Soloveichik, E. Galstyan and G. Majkic, Enhanced critical currents in $(\mathrm{Gd}, \mathrm{Y}) \mathrm{Ba} 2 \mathrm{Cu} 3 \mathrm{Ox}$ superconducting tapes with high levels of $\mathrm{Zr}$ addition, Supercond. Sci. and Tech., 26 (2013), 035006

[8] ed. S. E. Doyle, History of Liquid Rocket Engine Development in the United States 1955-1980, San Diego: Univelt, 1992, Part 3 Ch. 4, 69-122

[9] C. P. Bean, Magnetization of hard superconductors, Phys. Rev. Lett., 8 (1962), 250

[10] E. H. Brandt and M. Indenbom, Type-II-superconductor strip with current in a perpendicular magnetic field, Phys. Rev. B, 48 (1993), 12893

[11] N. Amemiya, Z. Jiang, Y. Iijima, K. Kakimoto and T.Saitoh, Total AC loss of $\mathrm{YBCO}$ coated conductor carrying $\mathrm{AC}$ transport current in $\mathrm{AC}$ transverse magnetic field with various orientations, Supercond. Sci. Technol. 17 (2004), 983-988 